\title{
Simulation of Breathing Patterns and Classification of Sensor Data for the early detection of impending Sudden Infant Death
}

\begin{abstract}
In this work, a methodology for the classification of breathing patterns in order to prevent sudden infant death (SID) incidents is presented. The basic idea is to classify breathing patterns which might lead to SID prior to an incident. A thorax sensor is proposed, which is able to simulate breathing patterns given by certain parameters. A sensor combination of conductive strain fabric and an inertial measurement unit is used for data acquisition. The data is then classified using a neural network.
\end{abstract}

Keywords: Wearable sensors, SID, pattern classification, artificial neural networks, inertial measurement units

https://doi.org/10.1515/cdbme-2019-0101

\section{Introduction}

Sudden infant death is one of the most common causes of death of infants [1, 2]. In 2015, all in all 2.405 infants died in Germany, among those 127 died due to sudden infant death [3].

Sudden infant death (SID) is defined as death of an infant under the age of one year for an unknown reason. SID occurs unexpected during the sleep. Until today, physicians haven't found a certain cause for sudden infant death $[4,5]$. Nevertheless, some risk factors that are linked to SID have already been identified: sleeping on the stomach, overheating during sleep and smoking during pregnancy [2]. Due to the knowledge of these risk factors, the cases of SID have declined. Nevertheless, the sudden infant death remains the most common cause of infants dying in the first year $[1,6]$. In order to be able to predict and to prevent SID, the knowledge about vital signs which change right before the death is required. Poets et al. [7] published vital signs of nine infants died due to sudden infant death syndrome. Here infants were monitored in a clinical environment right before the incident. The monitoring parameters comprised heart rate

\footnotetext{
* Corresponding author: Michael Munz: University of Applied Sciences Ulm, Germany, Michael.munz@thu.de
}

as well as breathing frequency and breathing pattern. The results of this study showed, that the breathing pattern changes to gasping or to apnea right before the death. Scholle et al. [8] also described a correlation between long or high frequent apneas and the sudden infant death.

Furthermore, the relation between pathologic breathing patterns and the sudden infant death has already been discussed in the literature (e.g. [9]. It is concluded, that the gasping such as long or high frequent apneas indicate a risk factor for the sudden infant death.

Based on this knowledge, the aim of this work is to develop a monitoring device for observing the breathing patterns of an infant in order to detect an impending incident and to prevent SID. For this, a wearable chest belt sensor is developed for capturing chest movement during breathing, fusing both inertial measurement unit (IMU) and strain fabric data. Furthermore, a machine learning algorithm is used for classifying the breathing pattern by the captured chest movement and to predict SID incidents.

\section{Related work}

Several research groups have already developed devices to monitor the vital signs of infants. Most of these devices were developed for the use at home.

A webcam based tracking of the thorax movements is published by Olvera et al. [5] and Fang et al. [10]. Both systems detect apneas by using image processing algorithms. By using a camera, the chest movements can be monitored non-invasively.

Liniti et al. [11] describe a vest for monitoring the infants' vital parameters. Within the vest there are custom made dry Electrocardiography (ECG) electrodes. Additionally, two resistive strain sensors were integrated in the vest to measure the chest and abdominal breathing. Further, a temperature and moisture sensor are mounted to acquire more vital parameters.

Ferreira et al. [6] combine ECG electrodes, an accelerometer and a thermometer in a chest belt. and Fonesca et al. [12] describe a camera to monitor the skin pigmentation in order to observe the current hear rate. Further, an accelerometer is 


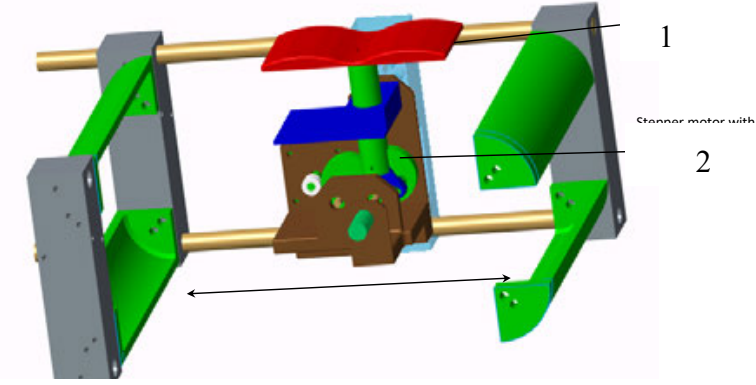

Figure 1: $C A D$ model of the mechanical thorax simulator with partial chest wall (1) and stepper motor with gear and crank drive (2).

used to acquire the infants' position. For detecting the breathing rate, a pressure sensor is used. Both groups describe algorithms which compare the vital parameters against thresholds for generating alarms.

Although multiple devices have been developed to prevent the sudden infant death, all the technologies seem to be circuitous to use. Due to the use of electrodes or the use of stiff chest belts, the infant could be affected during the night. In contrast to this, we propose in this work a comfortable chest belt with a low mechanical resistance, which does not affect breathing movements. The chest movements are recorded and further classified using a multi-layer neural network. The classifier is used to predict impending incidents based on long duration apneas such as gasping.

\section{Material and Methods}

\subsection{Mechanical thorax simulator}

In order to generate sensor data of several different breathing patterns, a mechanical thorax simulator was developed, able to simulate different user-defined breathing patterns (see figure 1). Therefore, realistic sensor data can be acquired. A stepper motor combined with a crank drive operates the simulator. The stepper motor was operated in sixteenth part microstepping mode. The stepper driver was controlled by an Arduino Mega. Additionally, an absolute encoder was connected to control the position of the crank drive.

Between the stepper motor and the crank drive, a two-stage gear with a translation of 1:3 in each stage was used. The gear reduced to velocity at the crank drive and increased the moment from the stepper motor. For calibration, the relation between the angle of the stepper motor and the displacement of the thorax was recorded using an Optitrack 3D markerbased camera system.

Table 1: Breathing pattern used in this work

\begin{tabular}{llr}
\hline Publication & Breathing pattern & \multicolumn{1}{c}{ Duration } \\
\hline Beck et al. [13] & Normal Breathing & $30 \mathrm{~s}$ \\
& Apnea & $60 \mathrm{~s}$ \\
Hasenstab et al. [9] & Normal + short apnea & $110 \mathrm{~s}$ \\
Huch et al. [14] & Gasping & $10 \mathrm{~s}$ \\
Kahn et al. [15] & Normal Breathing & $10 \mathrm{~s}$ \\
& Apnea & $10 \mathrm{~s}$ \\
\hline
\end{tabular}

A database of different breathing patterns was created using polysomnography recordings out of different publications (table 1). The recordings from the chest movement were digitalized using the Engauge Digitalizer (http://digitizer.sourceforge.net/). The input parameters (angle and velocity of stepper motor) for the thorax simulator were calculated based on these patterns using a transformation algorithm and calibrated parameters.

\subsection{Breathing sensor}

The custom-made breathing sensor is based on a conductive strain fabric (EeonTex Eeonyx, Pinole USA). This fabric changes its electrical resistance when strain is applied. The changing resistance is measured using a wheatstone bridge in combination with an INA128 instrument amplifier. The amplified signal is filtered with an analogue RC low pass filter and again amplified using a non-inverting operation amplifier MCP 6001, to enable a higher signal/noise ratio. The amplified signal is digitalized on an Arduino Micro board with a built-in 10-bit A/D converter.

This fabric can be wrapped around the chest and can be fixed with a hook-and-loop fastener. During the in- and expiration, the change of circumference of the chest over time can be measured. Furthermore, an MPU 9250 3D inertial measurement unit (IMU) is attached to the chest belt to acquire the movements of the thorax in order to achieve redundant measurements. For validation, the measurements were compared with the records of a commercially available polysomnography device (Porti 7, Heinen + Löwenstein, Bad Ems, Germany). For this, a subject was equipped with both devices. Recordings were simultaneously taken with both sensors while the subject changed between normal breathing and a simulated apnea by holding its breath. 

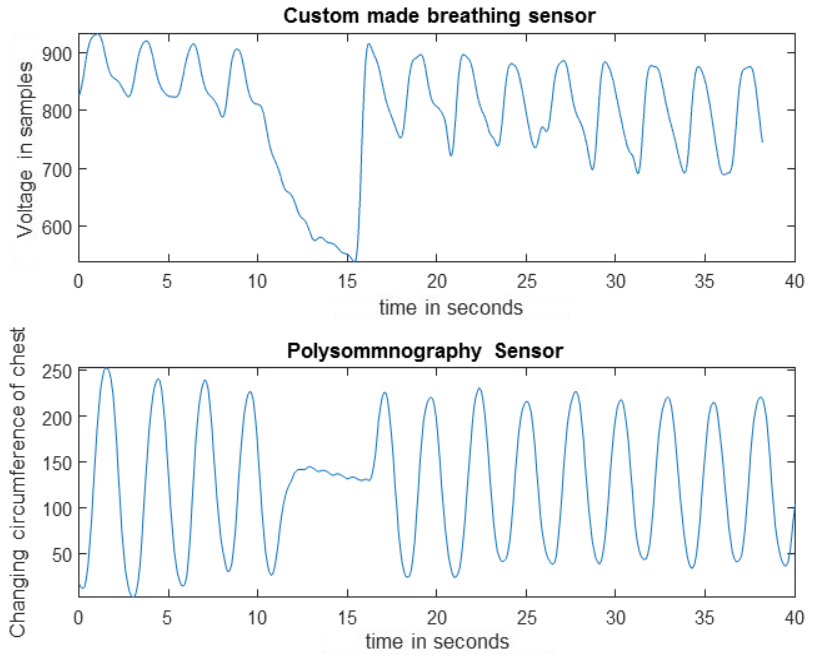

Figure 2: Data of the custom-made breathing sensor (top) compared with a polysomnography sensor (bottom) for one of the test series used for validation.

\subsection{Feature extraction and classification}

The thorax simulator was used to generate sensor data from different breathing patterns, provided in the literature as described above. Based on this data, a classification system was developed to show the fundamental feasibility of using the data for two classes were trained: healthy breathing (class 1) and pathological (class 2, covering abnea, grasping and any other pathological breathing prediction of impending incidents. For simplification, only two clag).

After pre-processing and filtering the raw data from both strain fabric sensor and IMU, the data is fused on feature level. First, a 3 second sliding window is applied to the time series data, overlapping of $50 \%$. From each time window, the following three features are extracted from the strain sensor data:

- Number of peaks (maxima and minima)

- Median of amplitude of consecutive peaks

- Mean of the slope of the signal

The following IMU acceleration based features are used:

- Median of the acceleration

- Sum of acceleration above threshold (here: $0.11 \mathrm{~m} / \mathrm{s}^{2}$ )

- Median of the envelope of the acceleration signal

Afterwards, those feature vectors are normalized to the range of $[-1,1]$, randomly shuffled and used for training a multilayer neural network. Training an evaluation was done using a 10-fold cross-validation. Cross entropy was used as performance metric. Parameter optimization revealed best results when using 12 neurons in the hidden layers.



Figure 3: Original breathing pattern (blue) compared with the movement from the chest wall simulator (red) in different breathing patterns (apnea (red area) and normal breathing).

\section{Results and discussion}

The validation of the thorax simulator showed a slightly delay of time and amplitude over the duration of a breathing pattern (figure 3). Nevertheless, every single breathing cycle was adequately captured in duration and amplitude. The thorax simulator can therefore be used to simulate different breathing patterns for sensor data generation.

The validation of our custom-made breathing sensor shows that normal breathing such as apneas can be detected. In figure 2, one of the test series used for validation of our custom-made breathing sensor is compared to the output of the polysomnography device. As it can be seen, each single breath as well as the short apnea are well captured by the sensor. Although, when stress on the sensor is static, a drift of the sensor value can be observed. This drift is very common in fabric sensors and can be compensated by application of a calibrated time-dependant offset function.

The 10-fold cross validation of the neural network leads to 10 sets of performance metrics. The deviation of those 10 sets is used to assess the reliability of the validation output. A boxplot of the validation output is shown in figure 4: true positive rate (TPR) of $89,3 \%$, true negative rate (TNR) of $91,5 \%$, false positive rate (FPR) of $1,7 \%$, false negative rate (FNR) of $8,5 \%$ and the overall classification rate of $89,9 \%$.

The output value of the neural network can be interpreted as a probability or can be used as a threshold for selecting the operation point of the classifier, adjusting the ratio between detection rate and false alarm rate. Therefore, a Receiver Operating Characteristics (ROC) analysis is also carried out. For this, the network is again trained using holdout with $75 \%$ training data, $10 \%$ validation data and $15 \%$ test data. 


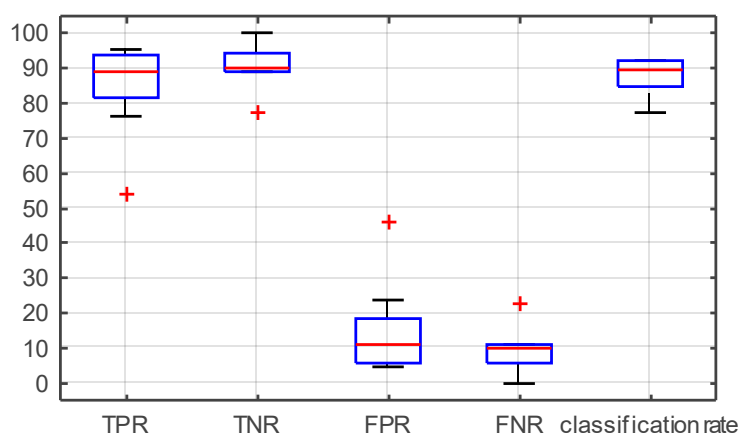

Figure 4: Boxplot of the validation results of the neural network: true positive rate (TPR), true negative rate (TNR), false positive rate (FPR), false negative rate (FNR) and the overall classification rate.

The thorax simulator was successfully applied on generating breathing data. Using this simulator, it is possible to simulate different situations and even critical breathing patterns. The patterns available in the literature can replicable be converted into simulation parameters. The validation of the sensor revealed promising results in comparison to polysomnography. The observed drift is well compensated by fusion data on feature level. The classifier applied as a first proof-of-concept shows good performance. Using different thresholds, the classifier can easily be applied to different requirements of false positive versus true positive rate based on the $\mathrm{ROC}$ results.

\section{Conclusion and Outlook}

The proposed thorax simulator for data generation in combination with available breathing pattern shows promising results for generation of critical and normal breathing patterns of infants. To the best knowledge of the authors, this is the first approach for simulation of breathing patterns for developing a pre-warn system for SID prevention. The combination of strain fabric sensor and IMU for capturing the breathing patterns is applicable to infant monitoring in order to prevent SID by detecting critical breathing patterns prior to apnea. The presented classification method is a very first approach, which has to be improved in future work, e.g. by applying time-sequence classification methods like Hidden Markov Models, recurrent neural networks or even majority votes of consecutive time window results. In addition to this, more breathing patterns should be integrated in the simulation step, as soon as available.

\section{Author Statement}

Research funding: The authors state no funding involved. Conflict of interest: Authors state no conflict of interest.

\section{References}

[1] F. R. Hauck and K. O. Tanabe, "International Trends in Sudden Infant Death Syndrome and Other Sudden Unexpected Deaths in Infancy: Need for Better Diagnostic Standardization," CPR, vol. 6, no. 1, pp. 95-101, 2010.

[2] T. Bajanowski and W. J. Kleemann, "Der plötzliche Kindstod," Rechtsmedizin, vol. 12, no. 4, pp. 233-248, 2002.

[3] Statistisches Bundesamt, Todesursachen bei Säuglingen: 2015. [Online] Available:

https://www.destatis.de/DE/ZahlenFakten/GesellschaftStaat /Gesundheit/Todesursachen/Tabellen/GestorbeneSaeuglin ge.html. Accessed on: Mar. 212017.

[4] H. C. Kinney and B. T. Thach, "The sudden infant death syndrome," (eng), The New England journal of medicine, vol. 361, no. 8, pp. 795-805, 2009.

[5] Diana Lucia Dorantes Olvera, David Santoyo López, Miguel Ángel Martínez Prado, Juvenal Rodríguez Reséndiz, Yerett, "Noninvasive monitoring system for early detection of apnea in newborns and infants," Biomedical Engineering and Sciences (IECBES), 2016 IEEE EMBS Conference on, pp. 494-498.

[6] A. Ferreira, Duarte Fernandes, Sérgio Branco, André P. Catarino, and Ana M. Rocha, "A Smart Wearable System for Sudden Infant Death Syndrome Monitoring," Industrial Technology (ICIT), 2016 IEEE International Conference on, pp. 1920-1925.

[7] C. F. Poets, R. G. Meny, M. R. Chobanian, and R. E. Bonofiglo, "Gasping and other cardiorespiratory patterns during sudden infant deaths," (eng), Pediatric research, vol. 45, no. 3, pp. 350-354, 1999.

[8] S. Scholle, S. Glaser, G. Zwacka, and B. Scheidt, "Diagnostik von Schlafapnoen-Möglichkeit zur SIDSPrävention?," Klinische Pädiatrie, vol. 202, no. 06, pp. 391395, 1990.

[9] K. A. Hasenstab and S. R. Jadcherla, "Respiratory events in infants presenting with apparent life threatening events: is there an explanation from esophageal motility?," (eng), The Journal of pediatrics, vol. 165, no. 2, 250-255.e1, 2014.

[10] Fang, Chiung-Yao and Hsieh, Hsin-Hung and Chen, SeiWang, "A Vision-Based Infant Respiratory Frequency Detection System," Digital Image Computing: Techniques and Applications (DICTA), 2015 International Conference on, pp. 1-8.

[11] C. Linti, H. Horter, P. Osterreicher, and H. Planck, "Sensory baby vest for the monitoring of infants," in International Workshop on Wearable and Implantable Body Sensor Networks (BSN'06), Cambridge, MA, USA, Apr. 2006, pp. 135-137.

[12] Â. M. Fonseca, E. T. Horta, S. Sendra, J. J. Rodrigues, and J. A. F. Moutinho, "A Sudden Infant Death Prevention System for Babies," 2014 IEEE 16th International Conference on e-Health Networking, Applications and Services (Healthcom), no. 525 - 530, 2014.

[13] S. E. Beck and C. L. Marcus, "PEDIATRIC POLYSOMNOGRAPHY," (eng), Sleep medicine clinics, vol. 4, no. 3, pp. 393-406, 2009.

[14] R. Huch, G. Raichle, S. Engelhardt, and B. Beck-Schimmer, Eds., Mensch, Körper, Krankheit, 6th ed. München: Elsevier Urban \& Fischer, 2011.

[15] A. Kahn et al., "Sudden infant deaths: Stress, arousal and SIDS," Early human development, vol. 75, pp. 147-166, 2003. 